\title{
Indoor Deployment of Low-Power Wide Area Networks (LPWAN): a LoRaWAN case study
}

\author{
Pierre Neumann, Julien Montavont and Thomas Noël \\ ICube laboratory (CNRS), University of Strasbourg \\ Strasbourg, France \\ Email: pierre.neumann@etu.unistra.fr, \{montavont,noel\}@unistra.fr
}

\begin{abstract}
The last decade saw the emergence of the Internet of Things (IoT) paradigm, which aims to connect any object to the Internet. In this context, a new type of wireless communication network emerged known as Low-Power Wire-Area Network (LPWAN). By contrast to well-known short range and multi-hop wireless networks, LPWAN networks allow long range communications at a low bit rate. Furthermore, LPWAN networks are considered to be integrated into 5G. Among LPWAN networks, the LoRaWAN technology gains more and more interest from the research and industrial communities. In this article, we have led a thorough experimental performance evaluation of LoRaWAN in an indoor environment. From this study, we quantify the limits of this technology and expose the merits of using LoRaWAN for IoT communications in the context of 5G.
\end{abstract}

Keywords-LoRaWAN; LPWAN; Internet of Things; Experimental Study;

\section{INTRODUCTION}

Fifth generation networks (5G) extend traditional largescale wireless deployments with next-generation technologies enabling new applications and use cases. The transition from $4 \mathrm{G}$ to $5 \mathrm{G}$ will take at least a decade or longer as network operators, infrastructure vendors and device manufacturers progressively implement those new technologies, while one or more standardized $5 \mathrm{G}$ technologies are being defined. During the transition period, several technologies will be investigated to potentially become key ingredients of 5G. In this context, a challenging issue for $5 \mathrm{G}$ is the support of the Internet of Things (IoT). According to Cisco, up to 50 billions of objects will be integrated to Internet by $2020{ }^{1}$. Such an integration raises challenging issues, ranging from scalability to security.

To address those challenges, one of the investigated technology by the 3rd Generation Partnership Project (3GPP) group is LoRaWAN [1]. LoRaWAN is a new long range and low power wireless communication technology. By contrast to the short range multi-hop communication model generally used in IoT networks [2], LoRaWAN returns to a one-hop communication model, similarly to cellular networks. Short range multi-hop wireless networks have been studied both intensively and extensively during the past few years. Still,

\footnotetext{
${ }^{1}$ http://www.cisco.com/c/en/us/solutions/internet-of-things/overview.html
}

serious scientific challenges remain such as the deployment complexity [3], the need of an energy consuming routing protocol [4] or the lack of security [5]. With the one-hop model introduced by LoRaWAN, the network architecture is simplified and resolves most of the issues encountered with multi-hop wireless networks. However, LoRaWAN introduces very limited transmission capabilities, that can not cope with all of the envisioned applications [2].

In this article, we present an in-depth empirical analysis of LoRaWAN performances over a real-world environment. The objectives of this study are twofold: (i) to explore the limits of networks using LoRaWAN, especially in terms of throughput, delay, energy consumption and packet delivery ratio; (ii) to investigate the merits of using LoRaWAN as an alternative for IoT communications in the context of 5G. Although other LPWAN solutions are available (e.g. SIGFOX), LoRaWAN offers several advantages: it is based on open standard (only the LoRa PHY is under license), allows for long-range and robust communications without requiring expensive components, and the consortium behind the technology (the LoRa Alliance) is open and develops a complete eco-system, ranging from residential to industrial types of network and applications [6]. This explains why we focus our study on LoRaWAN.

The rest of the article is organized as follows. The next section presents in a nutshell the wireless communication technology LoRaWAN. Section III gives an overview of LoRa alternatives for $5 \mathrm{G}$ and presents previous works based on LoRaWAN. Next, we present the testbed that we set up and used for all experiments followed by Section V where we present and analyze obtained results. Finally, conclusions and future work are presented in Section VI.

\section{LORAWAN BASICS}

LoRaWAN [1] is Low Power Wide Area Network (LPWAN) protocol that enables end-devices to wirelessly transmit and receive data at low-power. A LoRaWAN network is generally based on LoRa [7] which is a wireless modulation for long-range low-power low-data-rate applications developed by Semtech. LoRaWAN introduces three network entities: end-devices, gateways and a central network server. End-devices can only communicate with gateways 
via single-hop LoRa communication. On the other side, gateways are connected to the central server via the IP protocol suite. Figure 1 presents an overview of a LoRaWAN network.

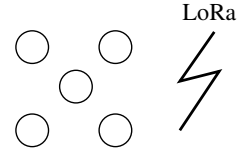

end-devices

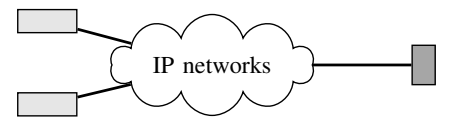

gateways network server
Figure 1. Example of LoRaWAN network

LoRaWAN introduces three modes of operation: Class A (the default), Class B and Class C (both optional). Those modes define how an end-device can access to the wireless medium. Although Class A allows for bidirectional communications, it focuses on upward traffic (i.e. from the end-devices to the network server). Class B and Class C introduce optional features for improving downward traffic (i.e. from the network server to an end-device). Class B and Class $\mathrm{C}$ are however not yet supported by the available hardware. In this article, we will therefore focus on Class A that all end-devices must support.

A Class A end-device schedules its transmission slots using an ALOHA-like method. Transmission slots are therefore scheduled regarding the application needs with small variations based on a random time basis. In addition, each transmission of an end-device is performed on a different radio channel in order to make the whole system more robust to interferences. In Europe, LoRa uses the $863-870 \mathrm{MHz}$ ISM band that is subdivided into channels. Three default channels (868.1, 868.3 and $868.5 \mathrm{MHz}$ ) are the minimum set that all network gateways should always be listening on. Additional channels can be dynamically configured by gateways. The parameters of such channels (e.g. center frequency, usable data rates, etc.) are transmitted to end-devices by control messages. Interferences are also mitigated by using different data rates (ranging from 0.3 to $50 \mathrm{kbps}$ ). Communications with different data rates do not interfere with each other.

Finally, in Europe, Class A end-devices should enforce duty-cycled limited transmissions to cope with the regulations of the ISM band defined by the European Telecommunications Standards Institute (ETSI). For this, channels are grouped in sub-bands. Whenever an end-device transmits a frame over a sub-band, it can not reuse this sub-band before $T_{\text {off }}$ seconds, $T_{\text {off }}$ being defined in Eq. 1 where TimeOnAir is the packet transmission time in seconds and DutyCycle is the defined duty cycle for the corresponding sub-band (usually $<1 \%$ by sub-band).

$$
T_{\text {off }}=\frac{\text { TimeOnAir }}{\text { DutyCycle }}-\text { TimeOnAir }
$$

For example, if an end-device takes $0.5 \mathrm{~s}$ to transmit a packet over a specific channel, the whole sub-band to which this channel belongs to will be unavailable for $49.5 \mathrm{~s}$. However, the duty cycle is defined on a per sub-band basis. If another sub-band is still available for this end-device, it can right away transmit a new frame over a channel that belongs to this very sub-band.

In Class A, downward communications (i.e. from a gateway to an end-device) are always initiated by enddevices. After each transmission, an end-device opens two reception windows - RX1 and RX2. RX1 is opened RECEIVE_DELAY1 $( \pm 20 \mu s)$ seconds after the end of its transmission slot. By default, RX1 uses the same frequency channel and data rate as the transmission slot. RX2 is opened RECEIVE_DELAY2 $( \pm 20 \mu s)$ seconds after the end of the transmission slot. By contrast to $\mathrm{RX} 1$, frequency channel and data rate used in RX2 can be negotiated between the gateway and the end-device. If a frame is received by the end-device during RX1, RX2 is not opened. RX1 and $\mathrm{RX} 2$ are long enough to efficiently detect a preamble. If a preamble is detected during $\mathrm{RX} 1$ (or RX2), the enddevice keeps its radio receiver active to receive the whole frame. RECEIVE_DELAY1 and RECEIVE_DELAY2 are respectively set to $1 s$ and $2 s$ for the $863-870 \mathrm{MHz}$ ISM band.

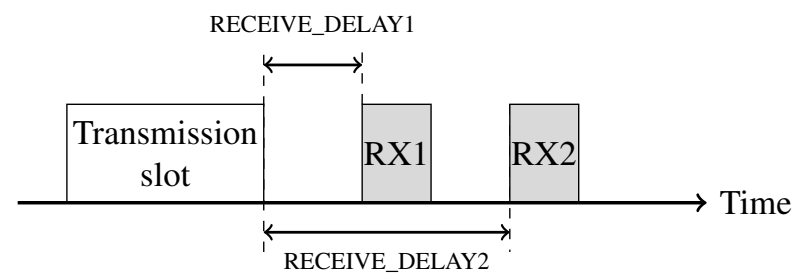

Figure 2. Transmission and reception slots in Class A

LoRaWAN introduces the concept of data rates corresponding to specific configurations of the physical modulation. Table I shows the available data rates for the 863$870 \mathrm{MHz}$ ISM band together with the equivalence between the data rate, the underlying configuration, the expected physical bit rate and the maximum allowed payload size. Data rate 0 to 6 use the LoRa modulation. Data rate 7 uses the Frequency-Shift Keying (FSK) modulation and is not considered in this article. The configuration is composed of the modulation type, the spreading factor (SF) and the bandwidth (BW) of the channel. This configuration directly impacts the time required to send a LoRa symbol (cf. Eq. 2) and therefore the time required to send a frame (i.e. TimeOnAir of Eq. 1). Lower data rates enable end-devices to communicate over longer distances at the cost of a longer time on air.

$$
T_{S y m}=\frac{2^{S F}}{B W}
$$




\begin{tabular}{|c|c|c|c|}
\hline $\begin{array}{c}\text { Data } \\
\text { Rate }\end{array}$ & $\begin{array}{c}\text { Configuration } \\
\text { (SF = Spreading Factor) }\end{array}$ & $\begin{array}{c}\text { Physical Bit } \\
\text { Rate (bps) }\end{array}$ & $\begin{array}{c}\text { Maximum Payload } \\
\text { Size (Bytes) }\end{array}$ \\
\hline 0 & LoRa: SF12 / 125 kHz & 250 & 51 \\
1 & LoRa: SF11 / 125 kHz & 440 & 51 \\
2 & LoRa: SF10 / 125 kHz & 980 & 51 \\
3 & LoRa: SF9 / 125 kHz & 1760 & 115 \\
4 & LoRa: SF8 / 125 kHz & 3125 & 242 \\
5 & LoRa: SF7 / 125 kHz & 5470 & 242 \\
6 & LoRa: SF7 / 250 kHz & 11000 & 242 \\
7 & FSK: 50 kbps & 50000 & 242 \\
\hline
\end{tabular}

Table I

LORAWAN DATA RATES

\section{RELATED WORK}

The LoRa wireless technology and the LoRaWAN standard being relatively recent, few references are available in the literature. In [8], the LoRa wireless technology is used to monitor the water level of troughs in order to allow cattlemen to remotely observe their livestock. Their outdoor test results showed that the location of the end-devices has a serious impact on the performance. Furthermore, the closer the enddevices are to the ground, the poorer is the transmission quality. They also showed that increasing the number of enddevices up to 100 decreases the Packet Delivery Ratio (PDR) by $17 \%$. However, this study was performed in the US ISM band that does not involve the same restriction than in the EU ISM band (no duty cycle limitation).

In [9], the authors present an outdoor performance evaluation of the LoRa wireless technology. The experimentations take place in Germany and use the $868 \mathrm{MHz}$ frequency. Two test cases were defined varying in modulation, bandwidth value and data rate. Obtained results showed that packets are successfully received at a distance of $6 \mathrm{~km}$ between the transmitter and the receiver when the payload size is limited to 10 bytes long. However, the Packet Error Rate (PER) increases along with the payload size. With a payload size of 50 bytes, nodes already experience a PER of approximately $10 \%$ for a $2 \mathrm{~km}$ distance. Unfortunately, some parameters used in this study are not compliant with the LoRaWAN standard (e.g. the bandwidth or data rate). Their findings are therefore difficult to extrapolate to a LoRaWAN environment.

The authors of [10] study how LoRaWAN suits for non-line-of-sight indoor operation. For this, they conducted an experimentation campaign using commercially available hardware in the campus of University of Oulu, Finland. This campaign included a single end-device attached to the arm of a researcher. The gateway antenna was installed at the University of Oulu antenna tower at height of $24 \mathrm{~m}$ from sealevel. Results showed that for most of the tested locations (the maximum distance between the end-device and the gateway was $420 \mathrm{~m}$ ), over $96 \%$ of the transmitted packets were successfully received. However, the authors only focus on the PDR and path loss. They do not take into account the energy consumption or test different data rate.

Finally, [11] focuses on a theoretical evaluation of the capacity and scalability of LoRaWAN. Results obtained via their analytic model showed that end-devices can benefit from an uplink channel of $2 \mathrm{kbps}$ at best. Obviously, this maximum throughput decreases with the distance to the gateway. In terms of scalability, they showed that a single gateway can potentially serve several millions of end-devices sending few bytes of data per day. However, most of the enddevices should be located in the vicinity of the gateway to achieve satisfactory performance.

As we can see and to the best of our knowledge, there is not yet an experimental indoor performance evaluation of LoRaWAN.

\section{EXPERIMENTATION SET UP}

LoRaWAN is envisioned as a key technology to support a large variety of Internet of Things applications. Among them, many scenarios (e.g. applications related to the smart factory) require an indoor network deployment. We therefore chose to focus our LoRaWAN performance evaluation in an indoor environment.

Our network is composed of one network server, one gateway and one end-device. We developed a minimal network server for the purpose of this evaluation. This server is in charge of decoding LoRaWAN frames forwarded by the gateway and log all measurements in a database.

The gateway is composed of one Raspberry Pi 2 connected to an IMST IC880A via the Serial Peripheral Interface (SPI) bus. Thus we have a standard Raspbian distribution with the LoRa gateway HAL and the packet forwarder code provided by Semtech. Thanks to the packet forwarder, the gateway communicates with the network server through the standard IPv4 / UDP stack. They are 2 kinds of communication, the upstream protocol and the downstream protocol. The upstream protocol is used when the gateway receives a RF LoRaWAN frame. The gateway then encodes the LoRaWAN frame in base64 and encapsulates it in a JavaScript Object Notation (JSON) structure with other parameters such as the timestamp or the Received Signal Strength Indicator (RSSI). Next, this JSON packet is forwarded to the network server which replies with an acknowledgement. The downstream protocol is used when the network server wants to communicate with an enddevice. For this purpose, the gateway periodically sends UDP packets that act as keep alive messages so an UDP port stays open (useful if the gateway is behind a NAT). Those packets are always acknowledged by the network server. The network server is then able to forward LoRaWAN frames to a gateway in a JSON structure. As for the upstream protocol, the JSON packet includes the LoRaWAN frame encoded in base64 and a value defining when this frame should be send by the gateway to fit in the receive windows and radio configuration. 


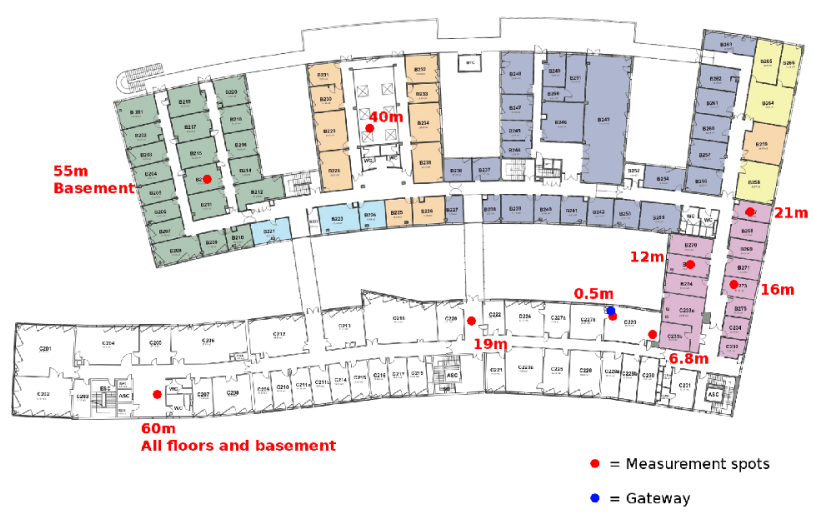

Figure 3. Gateway location and measurement spots in the building

The end-device is composed of one Raspberry Pi 2 which periodically sends commands to a RN2483 LoRa mote via the Universal Asynchronous Receiver/Transmitter (UART) interface. Those commands can be used either to configure the mote or to send LoRaWAN data. We only use the 3 default channels $(868.1,868.3$ and $868.5 \mathrm{MHz})$ of the EU $863-870 \mathrm{MHz}$ ISM Band. Each channel has a bandwidth of $125 \mathrm{KhHz}$ and belongs to the same sub-band. As the duty cycle of the sub-band is limited to $1 \%$ by ETSI, each channel is therefore limited to a duty cycle of $0.33 \%$. For the evaluation of the end-device energy consumption, we add a current sensor (Adafruit INA219 model) between the LoRa mote (being powered by the $3 \mathrm{~V}$ pin of the Raspberry Pi 2 ) and the Raspberry Pi 2. This sensor allows us to measure current consumption of the LoRa mote every $0.5 \mathrm{~ms}$ with a resolution of $0.1 \mathrm{~mA}$. The LoRa mote only supports Class A (cf. Section II) and therefore we only consider unconfirmed (i.e. no acknowledgments) upward traffic in our experiments.

Measurement spots are spread throughout the building on 13 different locations ranging from a distance of $50 \mathrm{~cm}$ from the gateway to $60 \mathrm{~m}$. Figure 3 shows the location of each measurement spot with their respective distance to the gateway. All measurement spots are located on the second floor of the building, at the same level than the gateway. In addition, the spot located at $60 \mathrm{~m}$ from the gateway includes 4 different measurement spots, one per floor (ranging from the basement to the 4th floor). Finally, the spot located at $55 \mathrm{~m}$ from the gateway is in the basement of the building.

\section{Performance evaluation}

The results presented in this section were extracted from our experimentation campaign. During the experiments, the end-device periodically sends 4 bytes of data encapsulated into a LoRaWAN frame. Due to the duty cycle limitation, the delay between two consecutive transmissions varies regarding the used data rate. Frames were spaced from 10 s to

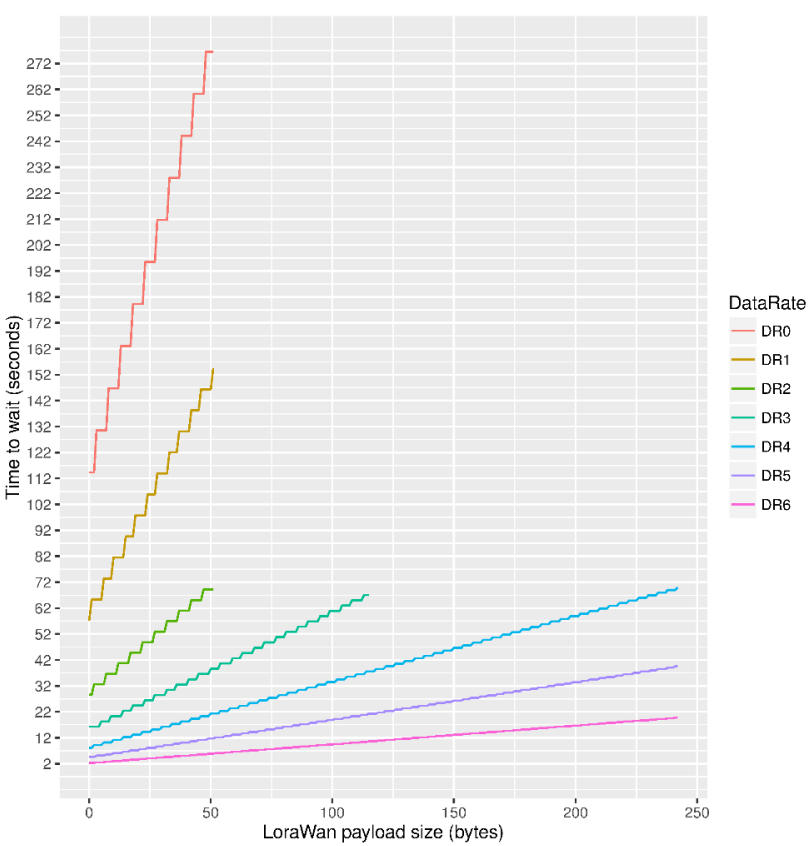

Figure 4. Time to wait for different LoRaWAN payload sizes at $1 \%$ duty cycle

a maximum of 2 minutes and 30 seconds to fit the regulation. For each spot we collected one-hour of data including the throughput, the Received Signal Strength Indicator (RSSI), the Signal to Noise Ratio (SNR), the Packet Error Rate (PER) and the current consumption. The whole set approximately represents 80 hours of measurements.

\section{A. Throughput}

The European regulation limits the default sub-band utilization to $1 \%$. This means that depending on the time on air used for a transmission, the device should wait for a sufficient amount of time so less than 1\% of the time is used by the device to send data on this sub-band. Regarding the selected data rate, frames of different sizes can take longer time on air to be transmitted [7].

Figure 4 shows the time to wait after the transmission of a frame for each payload size and data rate configuration. The LoRaWAN physical payload is composed of the MAC header, the MAC payload and a Message Integrity Check (MIC). This adds 13 bytes to the frame if no optional field are used. Such extra bytes are taken into account in our calculation. For readability reasons, we however chose to only represent the effective amount of data an application can send in Figure 4. In the worst case (i.e. with data rate 0 and the maximum packet size), an end-device should approximately wait for $4 \mathrm{~min}$ and $30 \mathrm{~s}$ before being able to initiate a new transmission. Even with no application data and data rate 0 , an end-device waits for almost $2 \mathrm{~min}$ between two consecutive transmissions. However, data rate 


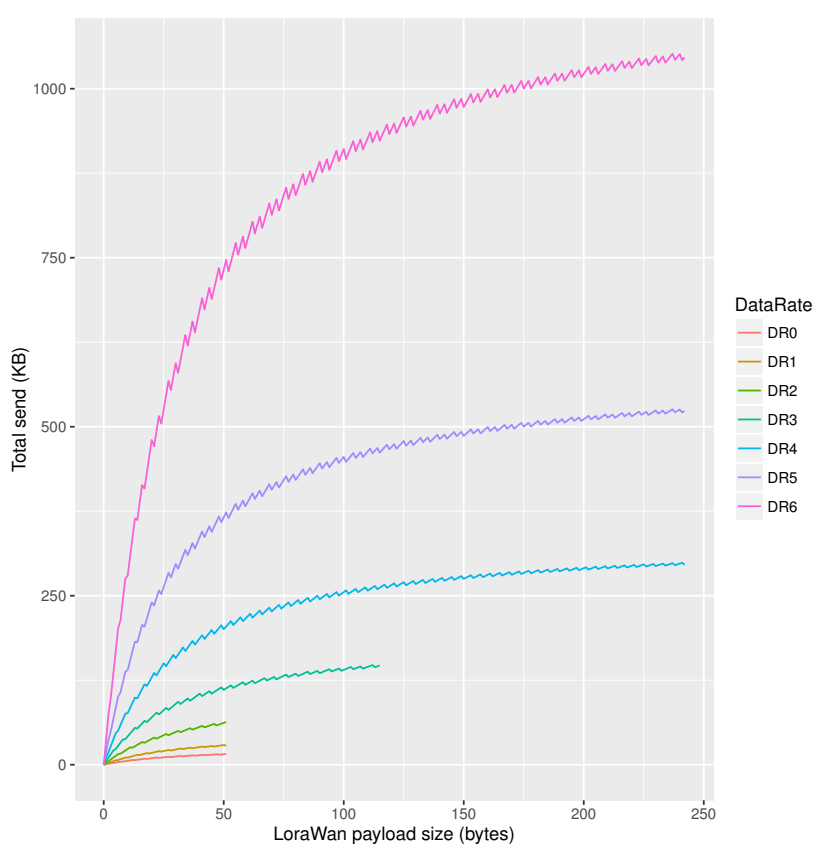

Figure 5. Total amount of data sent per day at $1 \%$ duty cycle

6 allows to reduce this delay to a minimum of $2 s$. Regarding the application needs, the data rate should be therefore carefully selected.

Figure 5 illustrates the maximum amount of data an enddevice can send per day over a single sub-band with a $1 \%$ duty cycle limitation. We can see that the data rate also limits the amount of transferred data. Data rate 6 allows the exchange of more than $1 \mathrm{MB}$ of data per day while this value drops to $15 \mathrm{~KB}$ with data rate 0 . As a result, data rate 6 seems to be the best configuration choice for both timesensitive and chatty applications. However, the different data rates also help to mitigate collisions between multiple enddevices. So configurations should ensure a good trade-off between meeting application needs and collision mitigation.

Since the 3 default channels are on the same sub-band, results illustrated in Figures 4 and 5 represent the limit of our network. The performance of a LoRaWAN network could be improved by increasing the number of sub-bands to increase the overall duty cycle. However, the gateway can listen only on a limited number of channels and those channels can not be spread over all possible values.

\section{B. RSSI and SNR}

Figure 6 shows the measured RSSI values for each data rate at each spot located at the same floor than the gateway. Generally, the RSSI quickly decreases in an indoor environment. Here, the RSSI decreases almost logarithmically with the distance from the gateway but remains good enough to successfully transmit data at $60 \mathrm{~m}$. In addition, it seems that the RSSI is not impacted by the data rate. So higher data

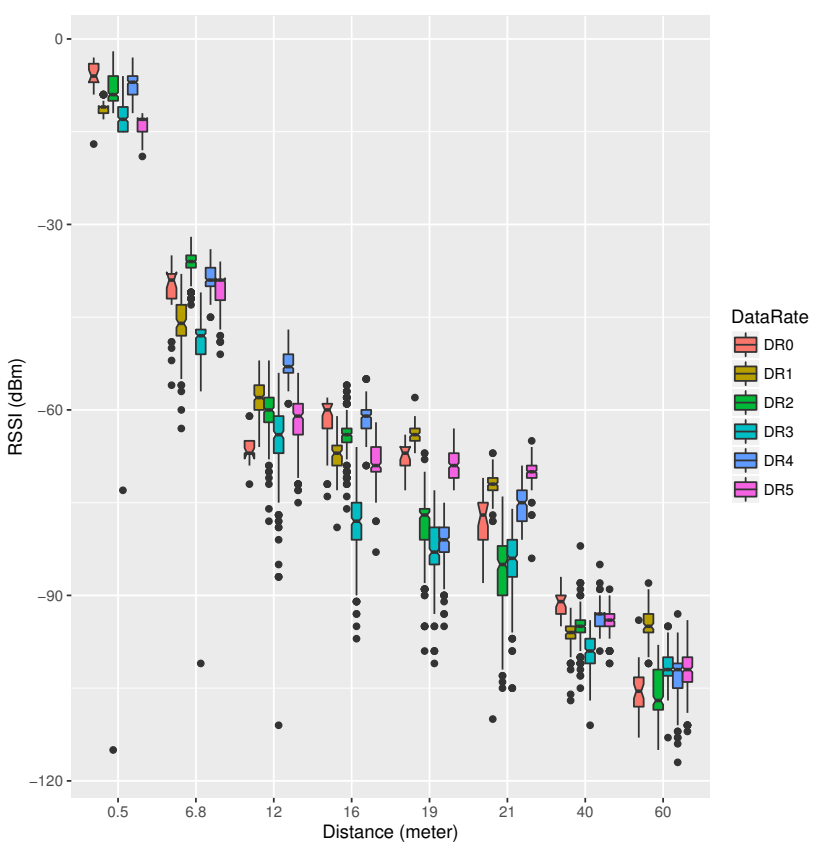

Figure 6. Measured RSSI values on 2nd floor

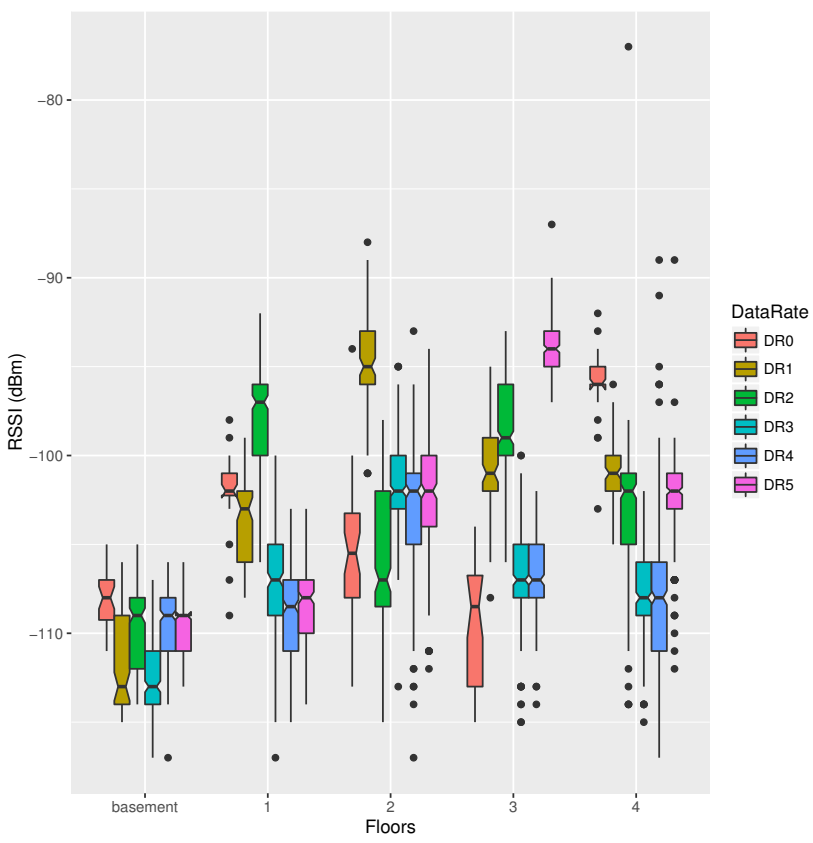

Figure 7. Measured RSSI values for each floor at $60 \mathrm{~m}$ 


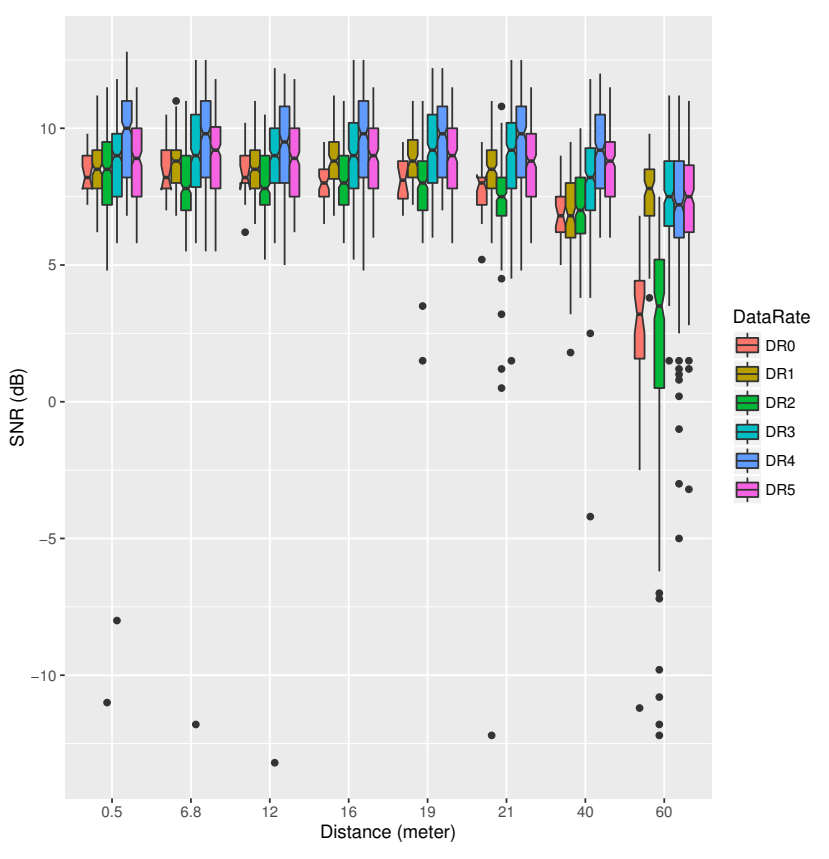

Figure 8. Measured SNR values on 2nd floor

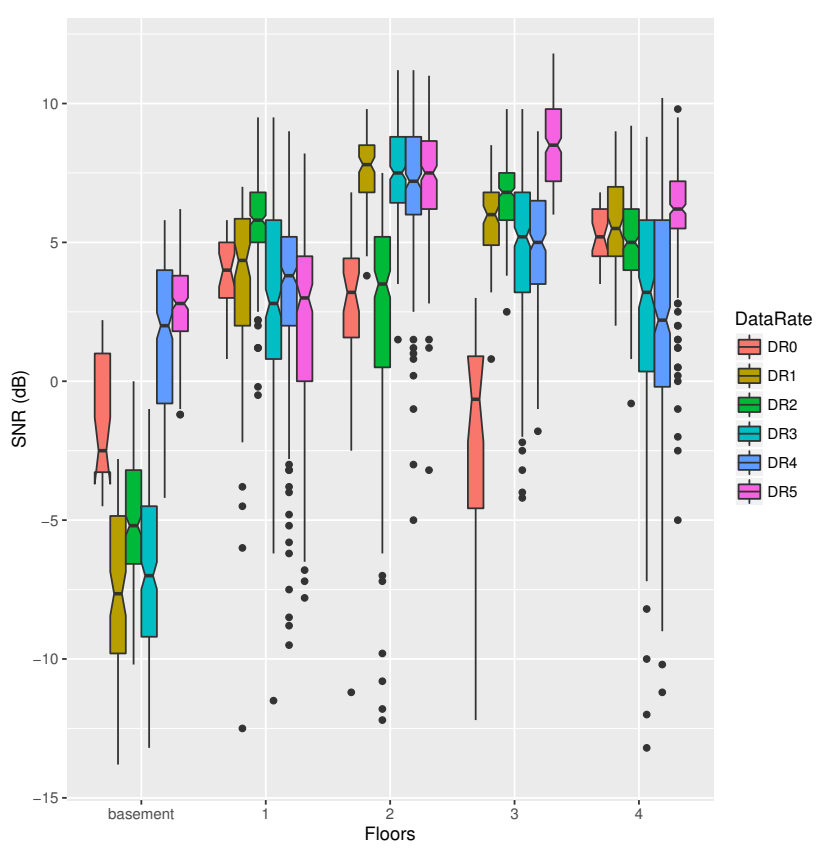

Figure 9. Measured SNR values for each floor at $60 \mathrm{~m}$

rates achieve better results in terms of bit rate and latency without sacrificing the connectivity coverage. Also, Figure 7 shows that the RSSI is not sensible to the floor location of the end-device, except for the basement.

By contrast, we can see on Figure 8 that the SNR remains constant while the end-device is located on the same floor as the gateway. Once the end-device moves to a different

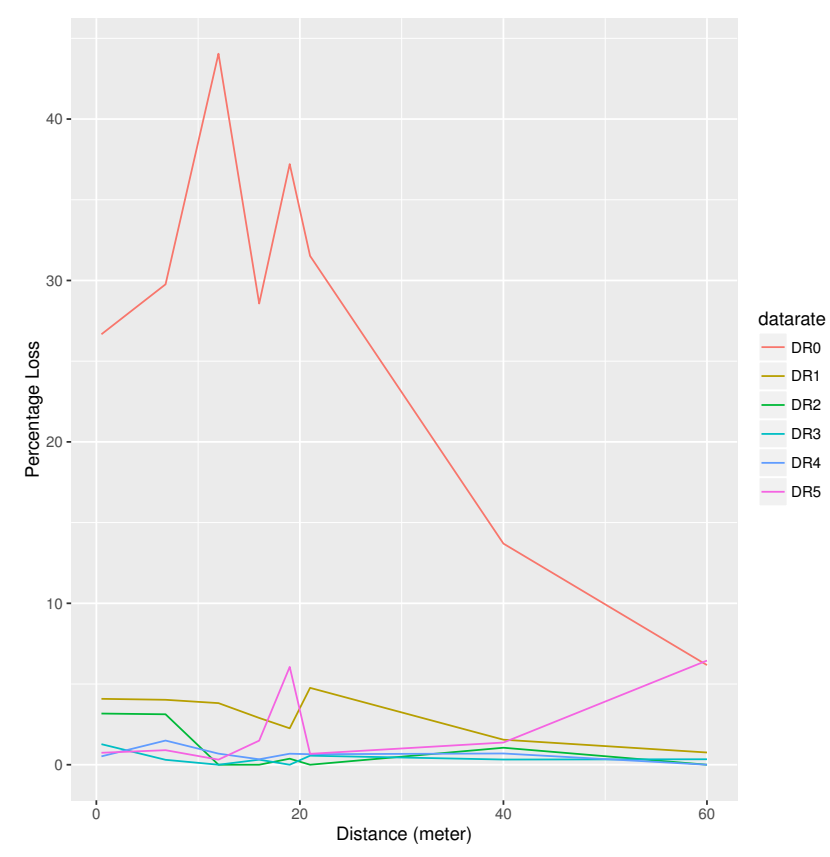

Figure 10. Percentage packet Loss on 2nd floor

floor, the SNR decreases whatever the data rate used as illustrated in Figure 9. The gateway experiences the worst SNR values when the end-device is located in the basement. As a result, when deploying a LoRaWAN network in a building, a specific attention should be paid on end-devices located in basements, as they might experience connectivity issues.

\section{Packet loss / Packet error}

When transmitting unconfirmed data, packet loss is an important indicator to quantify the network performance. For this purpose we distinguish packet error from packet loss. Packet loss refers to frames that are not received by the network server. Such frames can be either not received at all by the gateway or received by the gateway but with a bad CRC so they can not be decoded. Frames with bad CRC are what we called packet error. We observed in our experiments that, between multiple consecutive successful transmissions, frames can be duplicated and received with a bad CRC. Such phenomenon is more frequent with data rate 2 and when the end-device is close to the gateway.

Figure 10 shows the overall packet loss experienced by the gateway while the end-device moves on the 2nd floor. Surprisingly, the gateway experiences more than $25 \%$ of packet loss with the lowest data rate when the end-device is close to the gateway. Then, the packet loss decreases along with the distance to reach $0 \%$ when the end-device is located in the basement. By contrast, the packet loss experienced with data rate 5 increases along with the distance, up to $27 \%$ when the end-device is located on the basement. More 


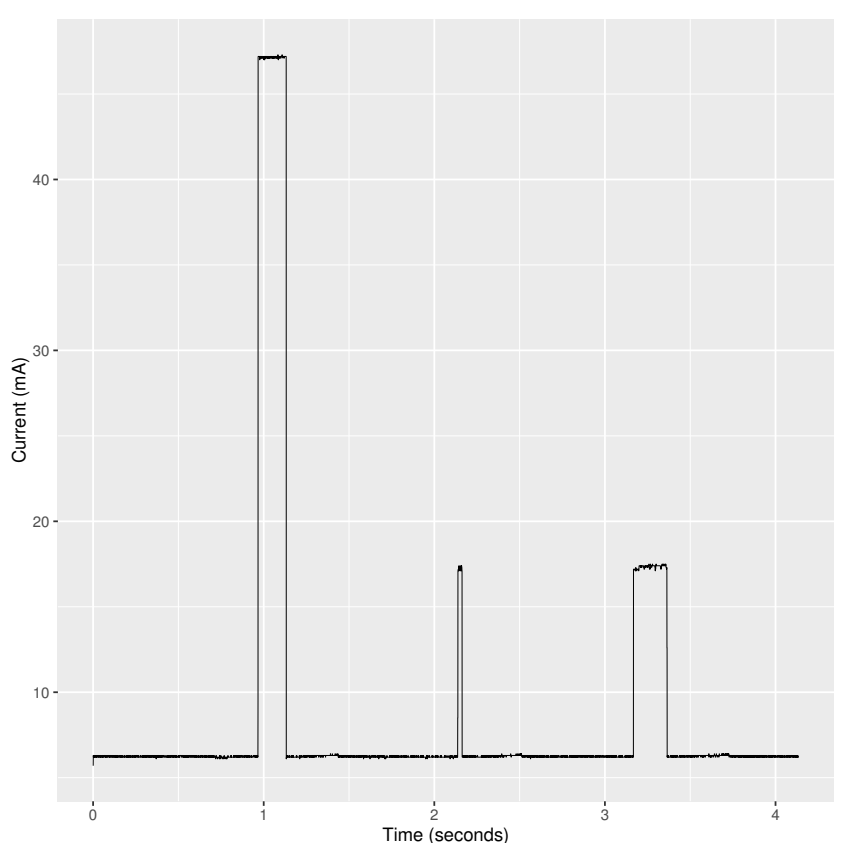

Figure 11. Current consumption for 4 bytes content at data rate 3

\begin{tabular}{|l|l|}
\hline \multicolumn{2}{|c|}{ At a typical tension of 3V } \\
\hline deep sleep & $9.9 \mu \mathrm{A}$ \\
idle & $2.8 \mathrm{~mA}$ \\
RX & $14.2 \mathrm{~mA}$ \\
TX $(14.1 \mathrm{dBm})$ & $38.9 \mathrm{~mA}$ \\
\hline
\end{tabular}

RN2483 CURRENT CONSUMPTION

generally, we can see on Figure 10 that with the other data rates the packet loss stays under $5 \%$ with some outliers on the same floor. This is why the right selection of data rate is needed to maximize the network performance.

\section{Power consumption}

The RN2483 data sheet gives the energy consumption values illustrated in Table II. Because the RN2483 is integrated on a mote, external factors can increase the energy consumption. Table III shows an average of the measured instantaneous current values at each steps of a transmission for each data rate. As we can see, the data rates do not impact the instantaneous current consumption.

Figure 11 shows the evolution of current consumption of the LoRa mote during the transmission of a 17 bytes LoRaWAN frame (4 bytes of data plus 13 bytes for headers and MIC) at data rate 3 with the maximum power $(14 \mathrm{dBm})$. We can clearly distinguish the transmission at about $47.5 \mathrm{~mA}$ followed by the two reception windows at $17.2 \mathrm{~mA}$. The transmission duration depends on the size of the frame and the data rate. By default, the first reception window (RX1) starts $1 s$ after the end of the transmission and the second

\begin{tabular}{|l|l|l|l|l|}
\hline sleep $(\mathrm{mA})$ & idle $(\mathrm{mA})$ & RX $(\mathrm{mA})$ & TX $(\mathrm{mA})$ & DataRate \\
\hline 3.4 & 6.2 & 17.2 & 47.8 & DR0 \\
3.4 & 6.2 & 17.2 & 47.9 & DR1 \\
3.4 & 6.2 & 17.2 & 47.4 & DR2 \\
3.4 & 6.2 & 17.2 & 47.6 & DR3 \\
3.4 & 6.2 & 17.2 & 47.5 & DR4 \\
3.4 & 6.2 & 17.2 & 47.5 & DR5 \\
\hline
\end{tabular}

Table III

LORA MOTE MEASURED INSTANTANEOUS CURRENT CONSUMPTION

\begin{tabular}{|c|c|c|c|}
\hline $\begin{array}{l}\text { Data } \\
\text { Rate }\end{array}$ & $\begin{array}{l}\text { Expected } \\
\text { Time }(\mu s)\end{array}$ & $\begin{array}{l}\text { Average Measured } \\
\text { Time }(\mu s)\end{array}$ & $\begin{array}{l}\text { Difference } \\
(\mu s)\end{array}$ \\
\hline 0 & 3351680 & 3588987 & 237307 \\
\hline 1 & 2692224 & 2929408 & 237184 \\
\hline 2 & 2362496 & 2599779 & 237283 \\
\hline 3 & 2197632 & 2434629 & 236997 \\
\hline 4 & 2125440 & 2362360 & 236920 \\
\hline 5 & 2084224 & 2321319 & 237095 \\
\hline
\end{tabular}

reception window (RX2) starts $1 s$ after the beginning of RX1. The different sizes of the two reception windows are related to the data rates. $\mathrm{RX} 1$ uses the same data rate as for the transmission which is in our case data rate 3 . RX2 always use by default data rate 0 . The LoRaWAN specifications define that even if no downward frames are received, the reception windows should open at least enough time to detect a preamble. Regarding our results, the reception windows are opened for 6 symbols. In addition to the transmission and reception states, the mote goes in sleep mode at $3.4 \mathrm{~mA}$ for few seconds.

Matching those values with the ones presented in Table III together with the data length, the data rate and the sending frequency, we are able to calculate the energy consumption of the end-device for all applications.

\section{E. Delay}

After sending a frame, a LoRaWAN end-device can not initiate a new transmission before the complete reception of a downward frame or before the end of RX2. Regarding the obtained energy consumption values, if no downward message is received, the parameters affecting the delay are the data length, the selected data rate and the serial link used to control the device. Table IV shows the measured delays between the moment just before the send of the command and when the mote acknowledge the effective transmission of the data. The expected time represents the time between the start of the transmission slot and the end of the second reception slot (RX2). The expected time does not take the serial communication into account. We can see that there is a constant delay of approximately $237 \mathrm{~ms}$ induced by the serial link. 


\section{CONClusions And Future Work}

In this article, we evaluated the performance of LoRaWAN unconfirmed uplink data frames in an indoor environment. We first showed the limitations in term of periodicity and size of data because of the ISM band regulation in a default channel configuration. Such regulation also limits the maximum amount of data that can be sent per day. Then, we evaluated the signal quality received from different locations, in order to verify the feasibility to cover an entire building with the LoRaWAN technology. The difference in the composition of walls between the rooms and the lab floors had not much impact on the quality of the transmission and packet loss. Only communications with the basement experienced degradations. In our experiments, a part of the basement is used as a parking space. Thus parking monitoring applications may take this kind of configuration into consideration. We also showed that the data rate can be a factor of loss and should be selected appropriately when configuring end-device. Finally, we showed the average current consumption of a LoRa mote and how the used data rate can impact the global energy consumption.

Depending on the network configuration, several data rates will not be able to fit specific application requirements such as the amount of data exchanged per a certain period of time. If an end-device is too far from the gateway, it will be constrained to lower data rate to maintain a satisfactory quality of transmission.

Encouraged by the results presented in this article, we plan to extend our performance evaluation. We will first focus on increasing the density of gateways to measure their impact on the network performance, especially the overall coverage and frame duplications. We will also increase the number of end-devices to evaluate the maximum capacity per gateway. Finally, the packet loss may be of crucial importance for specific applications. We will also take into consideration the performance of LoRaWAN confirmed uplink transmission (i.e. with acknowledgment).

\section{REFERENCES}

[1] N. Sornin, M. Luis, T. Eirich, T. Kramp, and O. Hersent, "LoRaWAN specification version 1.0," January 2015.

[2] S. Kraijak and P. Tuwanut, "A survey on IoT architectures, protocols, applications, security, privacy, real-world implementation and future trends," in proc. of the 11th International Conference on Wireless Communications, Networking and Mobile Computing (WiCOM 2015), September 2015.

[3] C. Zhu, C. Zheng, L. Shu, and G. Han, "A survey on coverage and connectivity issues in wireless sensor networks," Elsevier Journal of Network and Computer Applications, vol. 35, no. 2, pp. 619-632, 2012.

[4] J. Hao, B. Zhang, and H. Mouftah, "Routing protocols for duty cycled wireless sensor networks: A survey," IEEE Communications Magazine, vol. 50, no. 12, pp. 116-123, 2012.
[5] R. Roman, J. Zhou, and J. Lopez, "On the features and challenges of security and privacy in distributed Internet of Things," Elsevier Computer Networks, vol. 57, no. 10, pp. 2266-2279, 2013.

[6] L. Vangelista and A. Zanella and M. Zorzi, "Long-Range IoT Technologies: The Dawn of LoRa," Springer Lecture Notes of the Institute for Computer Sciences, Social Informatics and Telecommunications Engineering, vol. 159, pp. 51-58, December 2015.

[7] Semtech, "LoRa Modem Design Guide, rev.1," July 2013.

[8] Lukas, W. Tanumihardja, and E. Gunawan, "On the application of IoT: Monitoring of troughs water level using WSN," in proc. of the IEEE Conference on Wireless Sensors (ICWiSe), August 2015.

[9] M. Aref and A. Sikora, "Free space range measurements with Semtech Lora technology," in proc. of the 2nd International Symposium on Wireless Systems within the Conferences on Intelligent Data Acquisition and Advanced Computing Systems: Technology and Applications (IDAACS-SWS), September 2014.

[10] J. Petaejaejaervi, K. Mikhaylov, M. Hamalainen, and J. Iinatti, "Evaluation of LoRa LPWAN technology for remote health and wellbeing monitoring," in proc. of the 10th International Symposium on Medical Information and Communication Technology (ISMICT), March 2016.

[11] K. Mikhaylov, J. Petaejaejaervi, and T. Haenninen, "Analysis of Capacity and Scalability of the LoRa Low Power Wide Area Network Technology," in proc. of the 22th European Wireless Conference, May 2016. 